OPEN ACCESS

Edited by: Jing Yao,

Wuhan University, China

Reviewed by:

Kenneth E. Miller,

Oklahoma State University Center for

Health Sciences, United States

Yongchul Bae,

Kyungpook National University, South

Korea

*Correspondence:

Myeounghoon Cha

mhcha@yuhs.ac

Bae Hwan Lee

bhlee@yuhs.ac

Specialty section:

This article was submitted to

Pharmacology of Ion Channels and

Channelopathies,

a section of the journal

Frontiers in Pharmacology

Received: 17 August 2021 Accepted: 15 November 2021

Published: 08 December 2021

Citation:

Kwon M, Jung IY, Cha M and Lee BH (2021) Inhibition of the Nav1.7 Channel in the Trigeminal Ganglion Relieves

Pulpitis Inflammatory Pain.

Front. Pharmacol. 12:759730.

doi: 10.3389/fphar.2021.759730

\section{Inhibition of the Nav1.7 Channel in the Trigeminal Ganglion Relieves Pulpitis Inflammatory Pain}

\author{
Minjee Kwon ${ }^{1}$, Il Young Jung ${ }^{3}$, Myeounghoon Cha ${ }^{2 *}$ and Bae Hwan Lee $\mathrm{L}^{2,4 *}$ \\ ${ }^{1}$ Department of Nursing, Kyungil University, Gyeongsan, South Korea, ${ }^{2}$ Department of Physiology, Yonsei University College of \\ Medicine, Seoul, South Korea, ${ }^{3}$ Department of Conservative Dentistry and Oral Science Research Center, Yonsei University \\ College of Dentistry, Seoul, South Korea, ${ }^{4}$ Brain Korea 21 PLUS Project for Medical Science, Yonsei University College of \\ Medicine, Seoul, South Korea
}

Pulpitis causes significant changes in the peripheral nervous system, which induce hyperalgesia. However, the relationship between neuronal activity and Nav1.7 expression following pulpal noxious pain has not yet been investigated in the trigeminal ganglion (TG). The aim of our study was to verify whether experimentally induced pulpitis activates the expression of Nav1.7 peripherally and the neuronal activities of the TGs can be affected by Nav1.7 channel inhibition. Acute pulpitis was induced through allyl isothiocyanate (AITC) application to the rat maxillary molar tooth pulp. Three days after AITC application, abnormal pain behaviors were recorded, and the rats were euthanized to allow for immunohistochemical, optical imaging, and western blot analyses of the Nav1.7 expression in the TG. A significant increase in AITC-induced pain-like behaviors and histological evidence of pulpitis were observed. In addition, histological and western blot data showed that Nav1.7 expressions in the TGs were significantly higher in the AITC group than in the naive and saline group rats. Optical imaging showed that the AITC group showed higher neuronal activity after electrical stimulation of the TGs. Additionally, treatment of ProTxll, selective Nav1.7 blocker, on to the TGs in the AITC group effectively suppressed the hyperpolarized activity after electrical stimulation. These findings indicate that the inhibition of the Nav1.7 channel could modulate nociceptive signal processing in the TG following pulp inflammation.

Keywords: Nav1.7 channel, pulpitis, pain, endodontics, trigeminal ganglion

\section{INTRODUCTION}

Pain is an intricate phenomenon, and orofacial pain, which is a multifactorial experience, includes distressing sensory response and emotional modality (Estrela et al., 2011; Schuh et al., 2019). Dental pain associated with pulp inflammation, known as pulpitis, is quite common, and ectopic persistent pain and hyperalgesia are frequently observed after pulpitis (Matsuura et al., 2013; Alelyani et al., 2020; Sooratgar et al., 2021). Although there have been many studies regarding the identification of pulpitis (Hargreaves, 2011; Rechenberg et al., 2016; Filippini et al., 2018), the fundamental mechanisms of pain initiation and maintenance in pulpitis are still not fully understood; however, they are associated with sodium channels in the trigeminal nerves. There are many origins of pain in the orofacial region; among them, the trigeminal nerve fiber, which is the primary nerve fiber, predominantly innervates the orofacial region. Pain sensation from the orofacial region is 
transmitted to the TG, which is composed of three main branches: the ophthalmic (V1), maxillary (V2), and mandibular (V3) nerve branches. Pulpitis is generally a consequence of injury to one or more branches (nearly V2 and/or V3) (Maarbjerg et al., 2017). Alterations in primary afferent nerve fibers result in increased excitability, which causes hyperalgesia (Byers and Närhi, 1999). These functional changes may be manipulated by specific signaling components in neurons (Sun et al., 2019). Ectopic neuronal activity triggered by increasing sensitization is coincident with changes in the expressions of several ion channels and receptors (Julius and Basbaum, 2001; Davies et al., 2006).

Voltage-gated sodium channels (VGSCs) are transmembrane protein complexes that play critical roles in the formation and conduction of action potentials in response to stimuli exceeding threshold values in neuronal cells (Hameed, 2019). Many studies have shown that VGSCs control nerve excitability, inclusive of action potentials (Padilla et al., 2007; Luo et al., 2010). Moreover, they play an essential role in the conduction of electrical excitability (Rogers et al., 2006; Bi et al., 2016; Liu et al., 2019; Sun et al., 2019). Previous studies have indicated that sensory neurons alter the expression of VGSCs after inflammatory lesions (Wood et al., 2004; Dib-Hajj et al., 2010). The respective isoforms exhibit distinct distributions in the nervous system and have a noticeable electrophysiological composition (Cummins et al., 2007; Luo et al., 2010). In particular, Nav1.7 is highly enriched in the dorsal root ganglion (DRG), TG, sympathetic ganglia, and nociceptors of the peripheral nervous system (Toledo-Aral et al., 1997; Luiz and Wood, 2016; Liu et al., 2019; McDermott et al., 2019). Various pain syndromes like erythromelalgia and paroxysmal extreme pain disorder are caused by aberrant Nav1.7 (Cummins et al., 2004; Choi et al., 2011). Thus, Nav1.7 performs a noticeable function in pain perception, particularly inflammatory pain (Emery et al., 2016; Bi et al., 2017). Previous studies have found that nociceptor-specific knockout of Nav1.7 abolishes inflammation-induced hyperalgesia (Julius and Basbaum, 2001; Yeomans et al., 2005; Zhang and Gan, 2017). Indeed, studies have demonstrated that a lack of Nav1.7 in sensory neurons leads to diminished hypersensitivity in inflammatory pain models (Minett et al., 2012; McDermott et al., 2019).

Considering that Nav1.7 is related to inflammatory pain, we hypothesized that inhibition of Nav1.7 in the TG could relieve the hyperalgesia in pulpitis. The present study aimed to investigate the mechanisms underlying allyl isothiocyanate (AITC)-induced pulpitis and Nav1.7 expression changes in the TG. Furthermore, this study could suggest the possible clinical treatments for severe painful dental diseases.

\section{MATERIALS AND METHODS}

\section{Animals}

Male Sprague-Dawley rats (200-220 g, Koatech, Pyeongtaek, Korea) were subjected to all experiments in the Laboratory
Animal Facility at Yonsei University, Seoul, Republic of Korea; these experiments were approved by the Institutional Animal Care and Use Committee (20190093). The animals were housed in temperature- $(22 \pm$ $2^{\circ} \mathrm{C}$ ) and humidity-controlled rooms $(50 \pm 10 \%)$ within plastic cages containing soft bedding in a 12-h light/dark cycle with food and water provided ad libitum. Rats were randomly assigned to one of the following three groups: the no pulp inflammation group (naive group; $n=12$ ), the pulp exposure with saline application group (saline group; $n=12$ ), and the pulp exposure with allyl isothiocyanate (AITC) application group (AITC group; $n=12$ ). All efforts were made to minimize animal suffering and reduce the number of animals used.

\section{Inflammation of Tooth Pulp}

The surgical procedure was performed as previously described (Cha et al., 2020). The rats were anesthetized using intraperitoneal (i.p.) sodium pentobarbital $(50 \mathrm{mg} / \mathrm{kg}$ ) before being placed in the supine position. The rat's mouth was gently opened to expose the pulp of the left maxillary first molar (M1) using a low-speed dental drill with a $2 \mathrm{~mm}$ round tungsten carbide bur. For rats in the AITC group, a small piece of dental paper point (diameter, $0.15 \mathrm{~mm}$; length, $20 \mathrm{~mm}$ ) soaked in AITC (Sigma-Aldrich, Milwaukee, WI, US) was applied for $2 \mathrm{~min}$ to the exposed M1 tooth pulp. In the saline group rats, the exposed pulp was treated similarly, while saline was applied instead of AITC. The exposed pulp cavity was then sealed with visible light-cured composite resin $\left(\mathrm{SDR}^{\circledR}\right.$, Dentsply Sirona, York, PA, US) using Singlebond Universal (3M ESPE, Maplewood, MI, US).

\section{Behavioral Testing}

On day 3 after AITC or saline administration to M1, behavioral tests were conducted to evaluate the intensity of the pulpitis-induced pain. Rats were habituated for $10 \mathrm{~min}$ to the test chambers, which consisted of a metal mesh floor under individual Plexiglas boxes $(9 \times 9 \times 18 \mathrm{~cm})$ on an elevated table. In this study, we aimed to evaluate the degree of pain by measuring the abnormal response of pain induced by pulpitis using four behavior measurement methods (Cha et al., 2020). The spontaneous face-grooming activity was monitored and videotaped for $10 \mathrm{~min}$. Different types of behaviors were assessed, including face-wash strokes, paw-licks, ear grasps, and chin rubs. All face-grooming actions were measured as separate events. For each observation session, the total duration of face-grooming events was evaluated. Videotaped behaviors were analyzed offline by assessors who were blinded to the group information of the rats.

\section{Tissue Collection and Preparation}

For immunohistochemistry (IHC), after the behavioral testing, the rats were perfused using $0.9 \% \mathrm{NaCl}$ and $4 \%$ paraformaldehyde in sodium phosphate buffer, and the maxillary bone and first molar were removed and post- 
fixed in the same fixative solution for $24 \mathrm{~h}$ and subjected to decalcification in $17 \%$ ethylenediaminetetraacetic acid (EDTA, pH 7.0 with addition of $\mathrm{NaOH}$ ) for 3 weeks. Additionally, the extracted ipsilateral TG specimens were collected and post-fixed overnight at $4^{\circ} \mathrm{C}$ before cryoprotection in $30 \%$ sucrose in phosphate-buffered saline (PBS, pH 7.4) for $24 \mathrm{~h}$.

\section{Immunohistochemistry}

To verify pulpal inflammation, tooth specimens were cryosectioned at $12-\mu \mathrm{m}$ intervals and stained with hematoxylin and eosin. Neutrophil infiltration was used as a criterion for pulpal inflammation. To identify the changes in TGs due to AITC-induced pain, the expression levels of Nav1.7 were assessed in the collected brainstems and ipsilateral TG specimens. Longitudinal sections of the TG specimens were obtained using a cryostat (Microm HM 525; Thermo Scientific, Walldorf, Germany). The collected tissues were blocked using $5 \%$ normal goat serum containing $0.1 \%$ Triton $\mathrm{X}-100$ for $1 \mathrm{~h}$ and then reacted with rabbit anti-Nav1.7 anti-serum (1:500; Cell Signaling Technology, Danvers, MA, United States). The sections were incubated for $2 \mathrm{~h}$ at $22 \pm 2^{\circ} \mathrm{C}$ with secondary antibodies consisting of biotinylated goat anti-rabbit IgG serum (1:200) and then washed with PBS and further incubated for $2 \mathrm{~h}$ with Alexa Fluor 488 and Cytm 3conjugated AffiniPure F (ab')2 Fragment Donkey Anti-Mouse IgG secondary antibodies (1:1,000; Jackson ImmunoResearch, West Grove, PA, United States). DAPI was used for counterstaining. Immunofluorescence images were obtained using an LSM700 confocal microscope (Zeiss, Oberkochen, Germany). Using the ZEN program (Zen 2.3 black software, Carl Zeiss), we set the reference threshold value for non-positive immunoreactive response, and the neurons selected above the threshold value were countered. The number of cells with colocalized expression of Nav1.7 was quantified. Three image stacks per rat ( $n=4$ rats/group) were used for image analysis, and standardized regions of interest were outlined in the TG sections to encompass neuronal cells of the maxillary branch (V2) of the trigeminal nerve at the intersection with the mandibular branch (V3) of the trigeminal nerve.

\section{Western Blot Analysis}

On three days post-injection, targeted TGs were collected, immediately frozen in liquid nitrogen, and stored at $-70^{\circ} \mathrm{C}$. TG explants were homogenized in lysis buffer (PRO-PREP; Intron Biotechnology, Pyeongtaek, Korea) containing phosphatase inhibitors (PhosSTOP; Roche, Mannheim, Germany). The supernatant was collected, and samples with the same amounts of protein were separated and transferred to a membrane (Merck Millipore, Darmstadt, Germany). The membrane was incubated with $5 \%$ bovine serum albumin solution for $1 \mathrm{~h}$ at room temperature and then incubated with anti-Nav1.7 antibody (ab65167, 1: 1,000; Abcam, Cambridge, United Kingdom) and $\beta$-actin antibody (no.4970, 1:10,000; Cell Signaling Technology) overnight at $4^{\circ} \mathrm{C}$. The membrane was then incubated with anti-rabbit horseradish peroxidase-conjugated secondary antibody (No. 7074, 1:10,000; Cell Signaling Technology) for $2 \mathrm{~h}$ at $20^{\circ} \mathrm{C}$. Proteins were visualized by applying a chemiluminescent substrate (GE Healthcare, Little Chalfont, United Kingdom) and observed using the LAS system (LAS 4000; GE Healthcare). $\beta$-Actin was used as a loading control.

\section{Optical Imaging With Voltage-Sensitive Dye for Neuronal Activity Recording in TGs}

Three days post-injection, the rats were anesthetized using urethane $(50 \mathrm{mg} / \mathrm{kg}$, i.p.). The dissected TGs were isolated in a optical camber. The chamber was filled with artificial cerebrospinal fluid (aCSF) equilibrated with a gas mixture (5\% $\mathrm{CO}_{2}$ in $\mathrm{O}_{2} ; \mathrm{pH}$ 7.4). The composition of the mock CSF was as follows (in $\mathrm{mM}$ ): $\mathrm{NaCl}, 126 \mathrm{mM} ; \mathrm{KCl}, 5 \mathrm{mM} ; \mathrm{CaCl}_{2}, 2 \mathrm{mM}$; $\mathrm{MgSO}_{4}, 2 \mathrm{mM} ; \mathrm{NaH}_{2} \mathrm{PO}_{4}, 1.2 \mathrm{mM} ; \mathrm{NaHCO}_{3}, 26 \mathrm{mM}$; and glucose, $30 \mathrm{mM}$. TGs were quickly isolated and prepared for optical imaging in the recording chamber. In brief, for staining, preparations were kept for $1 \mathrm{~h}$ in aCSF containing a voltagesensitive dye (VSD) (Di-2-ANEPEQ, $50 \mathrm{mg} / \mathrm{ml}$ in saline, Molecular Probes, Eugene, OR, US). After staining, the staining dye was exchanged with fresh aCSF solution. After $10 \mathrm{~min}$ of washing, optical imaging and data analysis were performed using a MiCAM02 hardware and software package (BrainVision, Tokyo, Japan). For optical imaging, we used a fixed-stage upright fluorescence microscope (BX51WI, Olympus, Tokyo, Japan) and a high-resolution MiCAM02 camera.

To record the activation of TGs, we used a halogen lamp $(150 \mathrm{~W})$. Light from the filtered tungsten-halogen lamp $(480-550 \mathrm{~nm})$ was reflected onto the surface of the TGs via a dichroic mirror. Fluorescence images were acquired using an absorption filter at a rate of $3.7 \mathrm{~ms} /$ frame (MiCAM02, BrainVision). The charge-coupled device-based camera captured a $4 \times 3 \mathrm{~mm}^{2}$ imaging area, consisting of $184 \times 124$ pixels. A concentric bipolar electrode was used for electrical stimulation of the TG. Twenty consecutive images in response to the electrical stimulation of V2 $(200 \mathrm{~ms}$ delay, $1 \mathrm{~ms}$ pulse width, $3 \mathrm{~s}$ interstimulus intervals) were averaged to reduce background noise and artifacts. Stimulation was performed with two intensities of 1 and $3 \mathrm{~mA}$. In order to observe the normal nociceptive responses, $1 \mathrm{~mA}$ applied in TG. And the hyperactive neuronal responses observed at $3 \mathrm{~mA}$. Image acquisition was triggered by an electrical stimulus. In oreder to verify the effects of Nav1.7 inhibition, ProTxII (selective Nav1.7 blocker, $1 \mu \mathrm{M}$, Tocris Bioscience, Bristol, United Kingdom) treated on TGs for $30 \mathrm{~min}$.

\section{Statistical Analysis}

The results of the behavioral tests and immunohistochemical parameters for each experimental group are expressed as the mean and standard error of the mean. An analysis of variance with a Student's t-test for post-hoc analysis was used to compare the differences in behavioral tests and immunohistochemical labeling (Nav1.7) in the TGs among the groups. The KruskalWallis test with Dunnett's test for post-hoc analysis was used to 

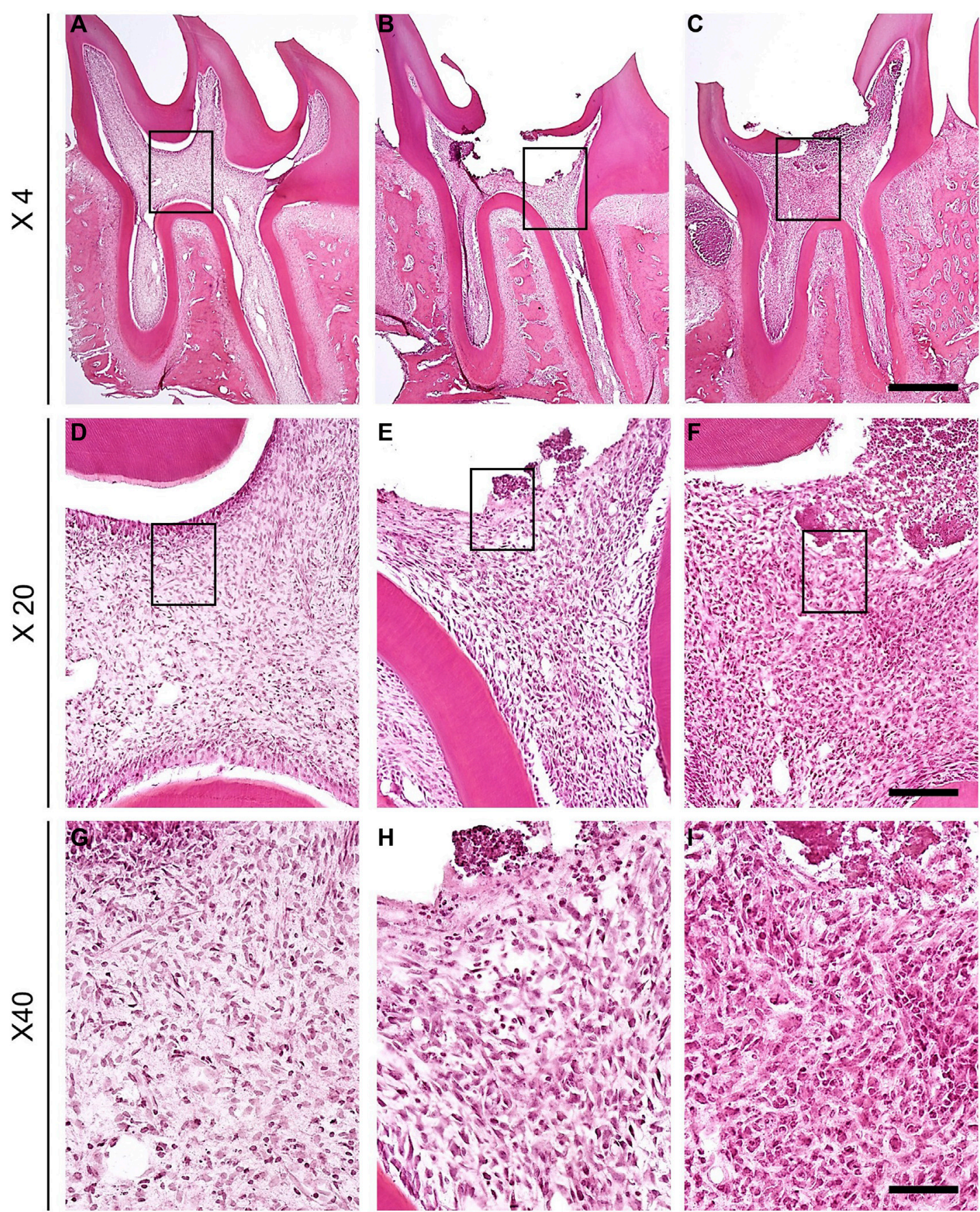

FIGURE 1 | Comparison of cross-section of naïve, saline-treated and molar exposed to AITC. Naïve (A), saline-injected (B), and AITC-injected (C) pulp tissues were compared (Scale bar $=200 \mu \mathrm{m})$. H\&E-stained sections of naïve, saline-treated, and inflamed dental pulp tissue verified the progression of inflammation. (D-F) Figures present enlarged black squares in (A-C) (Scale bar $=50 \mu \mathrm{m})$. (G-I) Figures indicate enlarged black squares in (D-F) $($ Scale bar $=10 \mu \mathrm{m})$. Figures indicate the AlTCinduced pulpal neutrophil changes.

compare immunohistochemical labeling (Nav1.7) in the brainstem among the groups. Statistical analyses were performed using SPSS software (version 23.0; IBM Corporation, Armonk, NY, United States). Statistical significance was set at $p \leq 0.05$. We excluded animals from the analysis when they showed any abnormality (poor general condition, loss of resin, or broken tooth during pulp inflammation) before or during the experiments. 
A

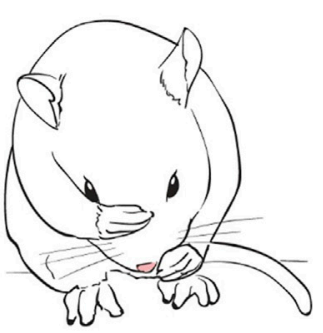

Face-wash strokes
B

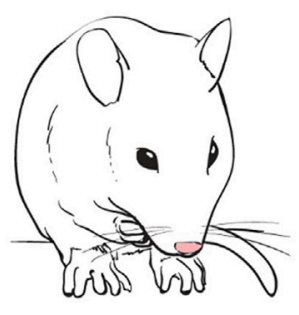

Chin rubs

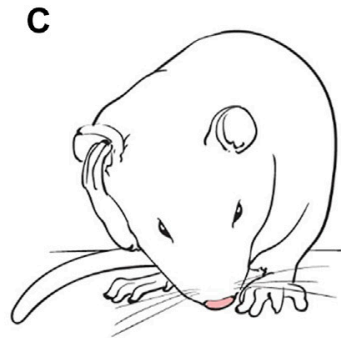

Ear grasps

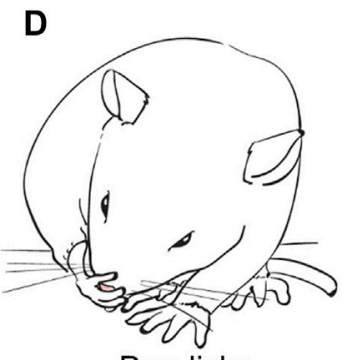

Paw-licks
E

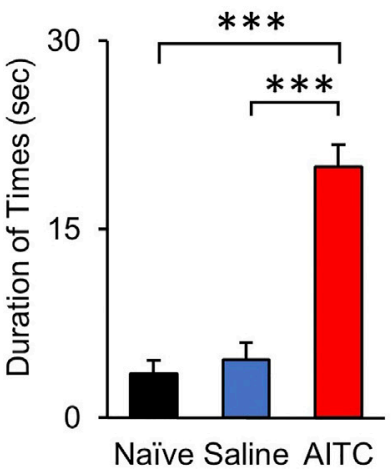

$\mathbf{F}$

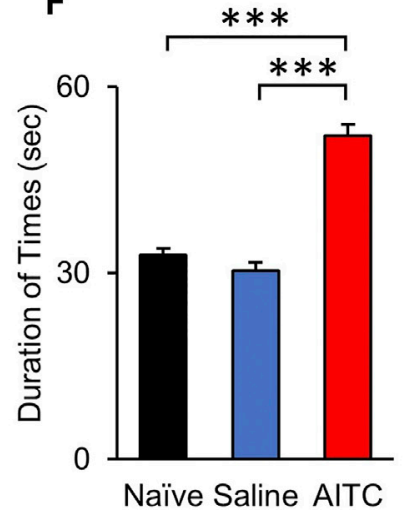

G

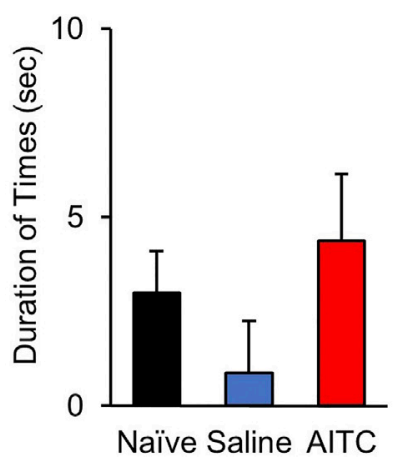

H

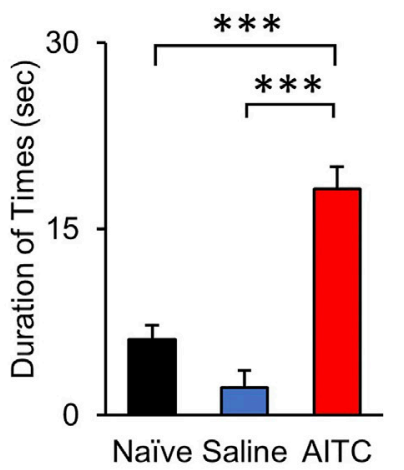

FIGURE 2|Behavioral analysis after AITC-induced pulpitis. (A-D) AITC-induced face grooming behaviors (face-washing, chin-rubs, ear grasps, and paw-licks) are illustrated and compared with naïve and saline-treated groups. Each graph show the duration time of grooming behaviors, face-washing (E), chin-rubs (F), ear grasps (G), and paw-licks (H). Histograms indicate the comparison of naïve, saline-, and AlTC-treated rats behaviors. Significant increases were observed in the durations of face-washing, chin rubbing, and paw licking in AITC-treated rats compared with naïve and saline-treated rats (Error bars represent SEM; ${ }^{\star \star \star} p<0.001, n=12$ each).

\section{RESULTS}

\section{Pulp Inflammation and Morphological Changes}

To determine whether dental pulp exposure with AITC injection led to pulpitis, hematoxylin and eosin staining was performed using a section of the first molar on the left maxillary bone. In the comparison of cross-sections of naïve (Figures 1A,D,G), salineinjected (Figures 1B,E,H), and AITC-exposed molars (Figures $\mathbf{1 C}, \mathbf{F}, \mathbf{I})$, histological signs of pulpitis were verified in the root pulp or periodontal tissue around the apex of AITC-treated group. This suggests that pulp inflammation can affect the radicular portion of the pulpal tissue.

\section{Assessment of Pain-Like Behaviors After Pulpitis}

To analyze the pain behavioral changes after AITC injection, face-grooming behaviors were compared among naïve, saline, and AITC-injected rats. Characteristic behaviors were analyzed by dividing them into face-washing, chin-rubbing, ear grasps, and paw-licking (Figures 2A-D) (Cha et al., 2020). Three days after the induction of AITC-induced pulpitis, increased facial grooming behaviors were noted in the AITC group rats compared with those in the naive and saline groups. In particular, a significantly longer duration of face-wash strokes was observed in the AITC group $(20 \pm 1.77 \mathrm{~s})$ than in the naive $(3.51 \pm 1.10 \mathrm{~s})$ and saline-treated $(4.62 \pm 1.37 \mathrm{~s})$ groups (Figure 2E). The durations of chin-rubbing (naive $32.87 \pm$ $4.09 \mathrm{~s}$; saline $30.37 \pm 9.54 \mathrm{~s}$; AITC $52.12 \pm 8.41 \mathrm{~s}$ ) and pawlicking (naive $6.12 \pm 2.23 \mathrm{~s}$; saline $2.25 \pm 0.99 \mathrm{~s}$; AITC $18.25 \pm$ $3.32 \mathrm{~s}$ ) were also significantly longer in the AITC group than in the naive and saline groups, thereby demonstrating a greater nociceptive response in rats treated with AITC (Figures 2F,H). The mean duration of ear grasping was longer than that in the naive $(3.01 \pm 1.53 \mathrm{~s})$ and saline groups $(0.87 \pm 0.39)$, albeit not significantly (Figure 2G). These results suggest that AITCinduced pulpitis causes orofacial pain and increased grooming behaviors.

\section{Expression of Nav1.7 in a Rat Model of Pulp Inflammation}

IHC was used to determine the cellular localization of Nav1.7 in rat TGs on day 3 after treatment. Representative IHC images of TGs from experimental groups showed that Nav1.7 was increased in the AITC-injected TGs compared to those in the naive and saline groups of TGs (Figures 3A-C). To analyze the expression of Nav1.7, 12 different images in each group were randomly selected, and Nav1.7-expressing neurons were counted. In the naive TGs, from a total of 1,507 neurons, only 118 showed Nav1.7 expressions $(8.02 \pm 1.25 \%)$. In addition, in the saline group, only 

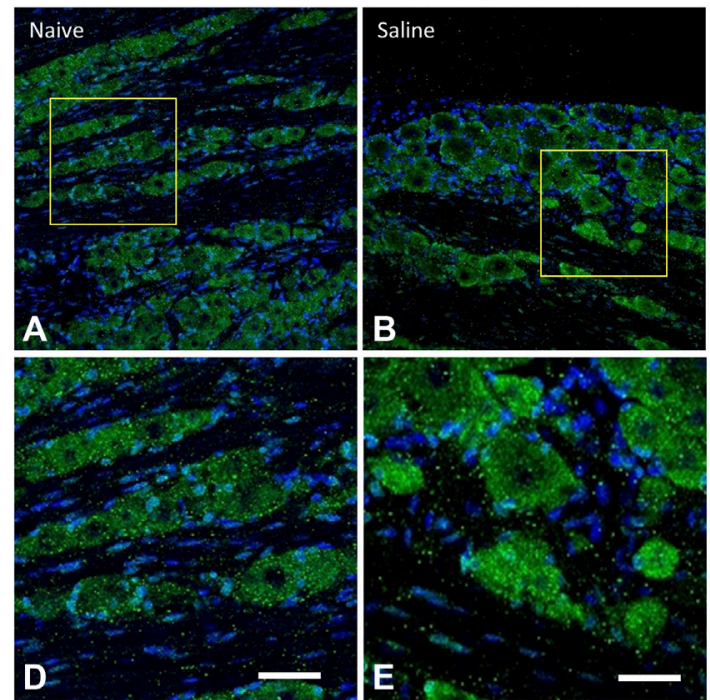

G

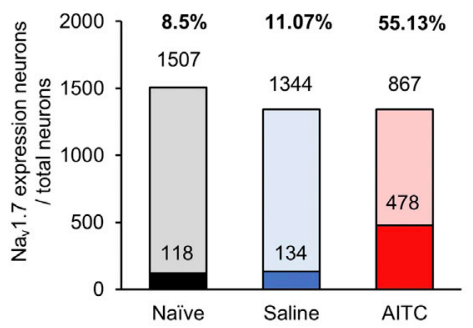

H

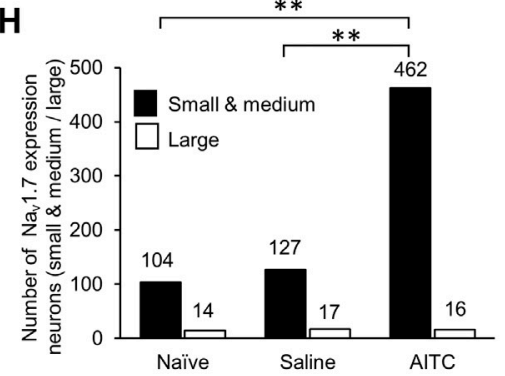

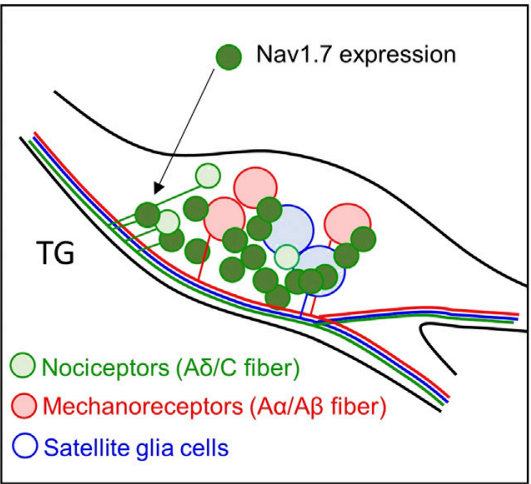

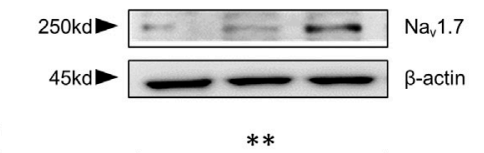

I

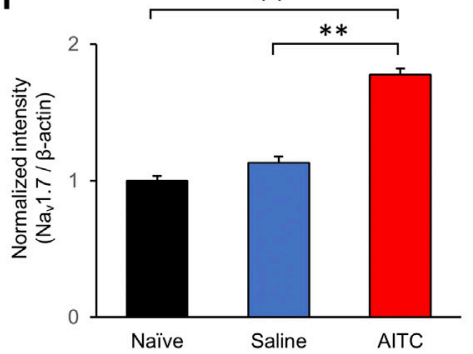

FIGURE 3|Expressions of Nav1.7 in the TG. (A-C) Immunostaining showing Nav1.7 expression in the TG. The protein expression of Nav1.7 increased in the TG in AITC-treated group. (D-F) Figures present enlarged yellow squares in a, b, and c, respectively. (G,H) The expression ratios were presented and the size-dependent Nav1.7 expression neurons were counted. The highest level of nav1.7 expression was observed in AlTC-treated groups. (I) Expressions of Nav1.7 were analyzed using western blot (Scale bar $=20 \mu \mathrm{m}$, Error bars represent SEM; ${ }^{* \star} p<0.01, n=4$ each).

134 out of 1,344 cells expressed Nav1.7 $(10.8 \pm 1.24 \%)$. However, in the AITC group, 478 neurons out of 1,344 expressed Nav1.7 $(35.23 \pm 4.61 \%)$. TG neurons were analyzed by size in our study, the results showed that Nav1.7 expressions were particularly concentrated in small- and medium-diameter $(\sim 45 \mu \mathrm{m}) \mathrm{TG}$ neurons (Small and medium/large size: Naïve, 104/14; Saline, 127/17; AITC, 462/16). These results showed a significant difference compared to the TGs of the naive and saline groups (Figures 3G,H). Additionally, to determine whether AITCinduced inflammation quantitatively increases Nav1.7 expressions, we analyzed the expression of Nav1.7 in TGs at the end of the experiment. Western blot analysis showed significantly increased Nav1.7 expressions in the AITC group (naive $1 \pm 0.03$; saline $1.13 \pm 0.04$; AITC $1.77 \pm 0.04$ ). These results suggest that the expressions of AITC-induced Nav1.7 could be observed in TGs.

\section{Comparison of Neuronal Responses in TGs}

In this study, we used VSD imaging to record membrane potential changes in rat TGs. To observe neuronal activity responding to electrical stimulation, we stimulated the V2 region of TGs and recorded the resultant TG neuronal activity. This allowed us to examine the spatial and temporal properties of TG responses to electrical stimulation. In the naive and saline-treated groups, VSD imaging revealed an electrical stimulus-evoked responses in the TGs by $1 \mathrm{~mA}$ stimulation (Figures 4A,B). In addition, we found pronounced neuronal activity in the AITC group (Figure 4C). Furthermore, under $3 \mathrm{~mA}$ stimulation, images showed higher activity patterns in the AITC group (Figures 4D-F). In comparison with 1 and $3 \mathrm{~mA}$, statistically significant increases in activation were observed at $3 \mathrm{~mA}$. The color-changed pixels of the different images were counted and compared (Figure 4G). In the comparison of activated pixel changes, 1 and $3 \mathrm{~mA}$ stimulation in the AITC group of the TGs showed significantly increased neuronal activity compared to the naive and saline groups (naive $1 \mathrm{~mA}: 329.66 \pm$ 73.93, saline $1 \mathrm{~mA}: 285.33 \pm 78.81$, AITC $1 \mathrm{~mA}: 1,275.62 \pm$ 291.74, naïve $3 \mathrm{~mA}: 848.5 \pm 66.83$, saline $3 \mathrm{~mA}: 525.33 \pm$ 101.91, AITC $3 \mathrm{~mA}: 2,428.8 \pm 384.75)$. The peak amplitude responses in the TGs are shown in Figure $\mathbf{4 H}$. In the comparison of peak amplitude changes, AITC-injected TGs showed significantly increased activity compared to naive and saline-treated TGs (naive $1 \mathrm{~mA}: 0.10 \pm 0.01$, saline $1 \mathrm{~mA}: 0.05 \pm$ 0.01 , AITC-treated $1 \mathrm{~mA} 0.18 \pm 0.03$, naive $3 \mathrm{~mA}: 0.15 \pm 0.05$, 

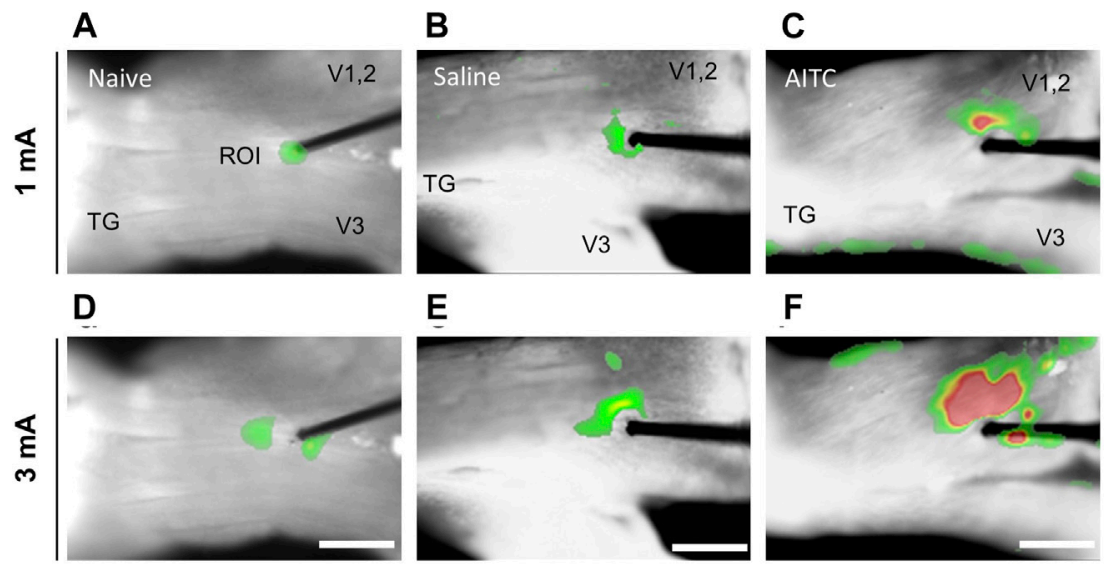

$\mathbf{G}$

$1 \mathrm{~mA}$

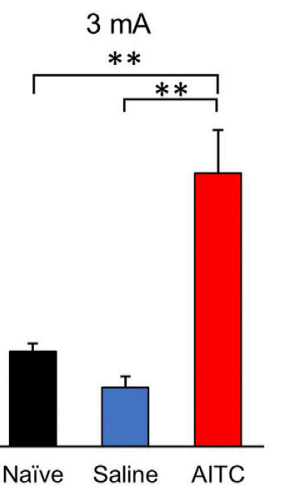

H

$1 \mathrm{~mA}$

$3 \mathrm{~mA}$
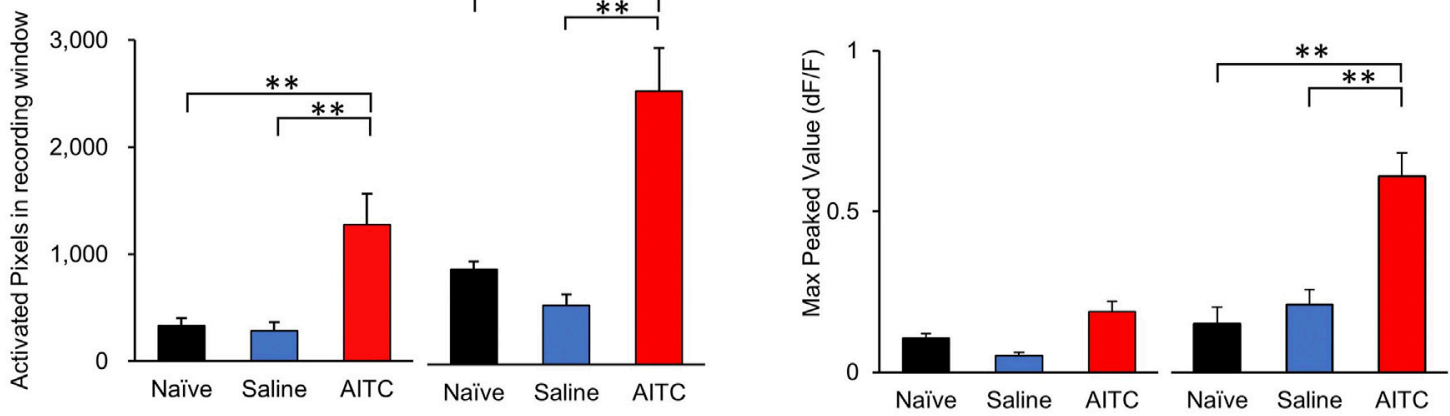

FIGURE 4 | Pulpitis-induced hyperactivities in TG. Comparison of optical signals at 1 (A-C) and $3 \mathrm{~mA}$ (D-F) stimulation in the naïve (A,D), saline-treated (B,E), and AITC-treated (C,F) TGs. In the AITC-treated TGs, stimulus-dependent enlarged optical signals were observed. (G) Activated pixels were analyzed in different intensity of stimulation. (H) Max peaked values after stimulation were analyzed. Compared to naive and sham, more activated pixcels and higher peak activity were observed in AITC-treated group (Error bars represent SEM; ${ }^{* *} p<0.01$ ).

saline $3 \mathrm{~mA}: 0.21 \pm 0.04$, AITC-treated $3 \mathrm{~mA} 0.60 \pm 0.07)$. Our data suggest that the AITC group of TGs had higher neuronal activity than the naive or saline group, and the difference was larger at high intensity.

\section{AITC-Induced Hyperalgesia is Inhibited by the Nav1.7 Channel Blocker ProTxII}

Optical imaging was performed in the TGs to elucidate the spatiotemporal dynamics of neural activity after ProTxII application. Electrical stimulation-induced neuronal activity of the TGs was observed (Figures 5A,B). Compared to $1 \mathrm{~mA}$ stimulation, higher amplitude stimulation $3 \mathrm{~mA}$ showed more pronounced and propagated neuronal depolarization in the TGs (Figures 5D,E). The excitatory signal amplitudes were significantly hyperexcited with increasing stimulus intensity, whereas only a slight increase was observed in the $3 \mathrm{~mA}$ stimulation with ProTxII (Figure 5C). Excitatory TG responses exhibit sustained neural activation, regardless of stimulus intensity. However, when a stronger stimulus was applied to the TGs, the peak value of the neural excitation increased and maintained (Figure 5F). Figure 5G shows the activation pixels before and after the application of ProTxII (1 mA AITC + vehicle: 1,425.51 \pm 109.36, $1 \mathrm{~mA}$ AITC + ProTxII: $752.42 \pm 185.97 ; 3 \mathrm{~mA}$ AITC + vehicle: $2,429.71 \pm$ 183.68, $3 \mathrm{~mA}$ AITC + ProTxII: $1,181.42 \pm 173.73)$. ProTxII inhibited the excitatory neuronal activity by less than $50 \%$ of the peaks amplitude of the optical signals compared with AITC only ( $1 \mathrm{~mA}$ AITC: $0.17 \pm 0.01,1 \mathrm{~mA}$ AITC + ProTxII: $0.08 \pm 0.01$; $3 \mathrm{~mA}$ AITC: $0.62 \pm 0.03,3 \mathrm{~mA}$ AITC + ProTxII: $0.22 \pm 0.05$ ) (Figure 5H). These results indicate that the increased neuronal activity generated by Nav1.7 expressions in AITC-treated TGs can be effectively inhibited by ProTxII.

\section{DISCUSSION}

Current research has focused on clarifying the pathogenesis of nociception following peripheral inflammation. In this study, we used AITC to induce an inflammatory response and confirmed the formation of acute pulpitis. Three days after the AITC injection, orofacial pain behaviors related to the inflammatory response were observed. AITC-induced pulpitis pain, as observed in our study, results in increased untypical behaviors such as a 

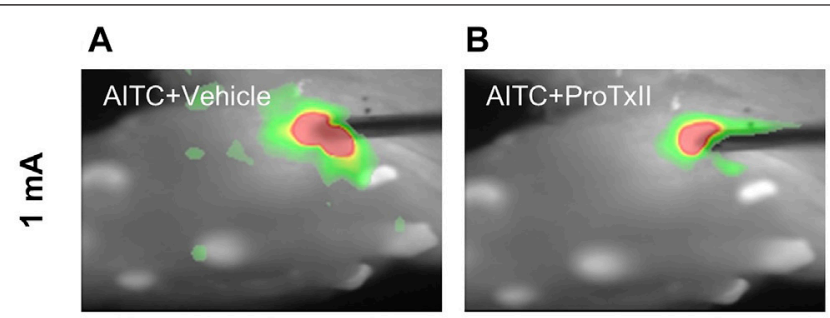

D

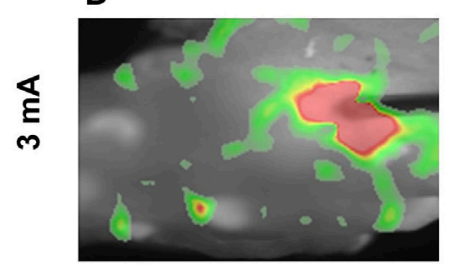

E

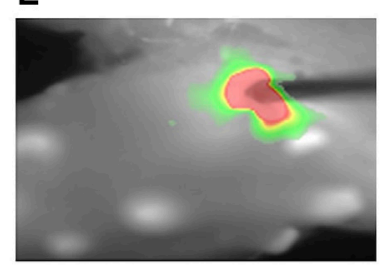

G

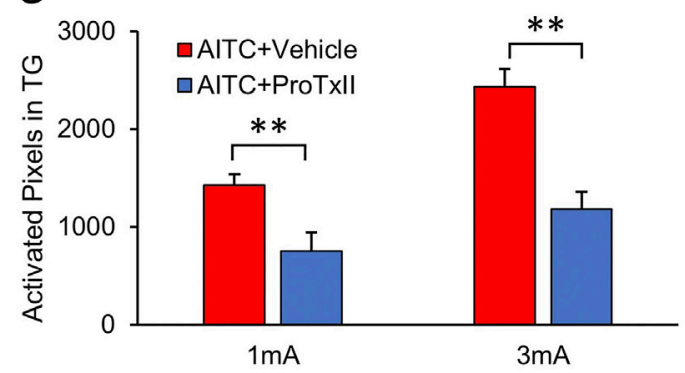

C

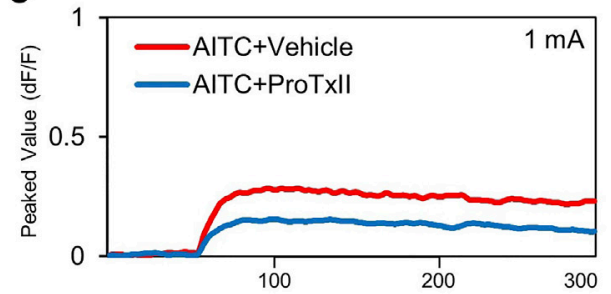

$\mathbf{F}$

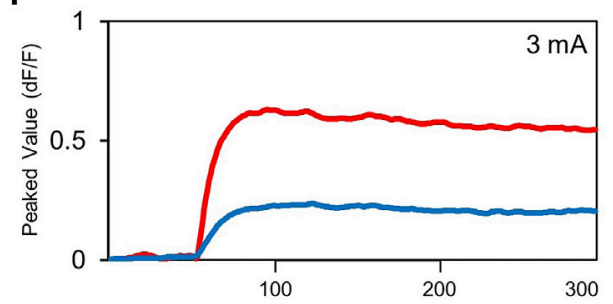

H

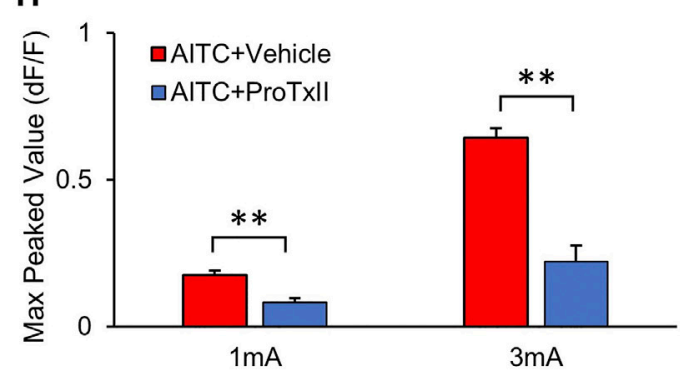

FIGURE 5 |Reduced neuronal activities in TG by application of the ProTxll. (A,B) Comparison of optical signals in the inhibition of Nav1.7 activation at 1 mA. (C,D) Comparison of optical signal changes after ProTxll at $3 \mathrm{~mA}$. Optical signals showed that the expanded and propagated neuronal activities were reduced after ProTxll. (E,F) Temporal peak value changes at 1 and 3 mA. (G) Comparison of the activated pixels in the TGs. (H) Comparison of the max peak values after ProTxll. Compared to AITC + Vehicle, significantly redeced activated pixcels and peak activity were observed in AITC + ProTxll group (Error bars represent SEM; $\left.{ }^{\star \star} p<0.01\right)$.

nociceptive reaction to the inflammatory response. The results of our study showed the upregulated expression level of Nav1.7 in the TGs during dental pulp inflammation. Moreover, ProTxII, a selective Nav1.7 inhibitor, suppressed the increased neuronal excitability following pulpitis. Therefore, the Nav1.7 channel could modulate nociceptive signal processing in the peripheral nervous system during pulp inflammation.

AITC has been used to examine pulp inflammation in animal models (Kawamura et al., 2010; Narita et al., 2012; Murano et al., 2021). Additionally, explicit abnormal behaviors were observed within 3 days of inflammation. Different analysis techniques can assess the level of pulpal induced-inflammation. The combination of several techniques is useful depending on the objective of the study. The observation of abnormal behavioral reactions, such as flinching, face-washing, chin-rubbing, ear grasps, paw-licking, and facial expressions, in rats has been widely used in several studies as a method of complementary pain assessment (Luccarini et al., 2006; Deuis et al., 2017; Sperry et al., 2018). Although it is difficult to establish an accurate meaning of abnormal behavior, increasing repetitive behavior, such as facial grooming, is considered a reliable symptom of intra-dental pain (Vos et al., 1998; Cha et al., 2020). Furthermore, alterations in voluntary movement and detection of abnormal behaviors following injuries have been recognized as indicators of pain in rats (Chudler and Byers, 2005). Our study demonstrated a significant increase in atypical behaviors such as face-wash strokes, chin rubs, and paw-licks in the AITC-induced pulpitis group, which is consistent with the results of previous study (Cha et al., 2020). As a nociceptive behavior to pulpitis, increased facial grooming appeared more often in the AITC-induced group than in the other groups. Taken together, increasing time spent on abnormal behaviors could be thought to be the result of pulpitisproduced pain.

Inflammatory pain in the oral cavity is induced by the initiation of plastic changes due to central or peripheral sensitization (Sessle, 2000; Tsuboi et al., 2011). Dental pulp inflammation induces central sensitization, which has been shown to increase subcutaneous nociceptive fields and abnormal activity (Chiang et al., 2002; Chiang et al., 2005; Kawamura et al., 2010). Furthermore, this inflammation causes respective receptors to activate intracellular signaling pathways, leading to increased membrane excitability of VGSCs (Amir et al., 2006; Hameed, 2019). Additionally, in the trigeminal nuclei, an increase in VGSCs implicated central sensitization, with higher strength in synaptic links and long-term potentiation (Siqueira et al., 2009). Hence, the role of VGSCs is involved in chronic pain such as inflammatory pain (Rogers et al., 2006; Dib-Hajj et al., 2010). Most subtypes that have been included in this pain 
pathway are Nav1.7, Nav1.8, and Nav1.9 (Fang et al., 2002; Luo et al., 2010). Among these channel subtypes, the expression of Nav1.7 increased in various chronic pain states (Dib-Hajj et al., 2007; Sun et al., 2019). Consistent with the results of previous studies, our investigation also confirmed that the expression level of Nav1.7 in TGs was upregulated in the pulpitis state. Nav1.7 is abundantly expressed in the DRG and sympathetic ganglion neurons in the peripheral nervous system (Sangameswaran et al., 1997; Toledo-Aral et al., 1997). Inflammation leads to the upregulation of Nav1.7 in DRG neurons, which project to the provoked region (Black et al., 2004; Gould et al., 2004; Strickland et al., 2008). The IHC results have shown that Nav1.7 is highlyexpressed in small diameter DRG in TG. Also, our data suggest that enhanced expression and function of Nav1.7 channels in TG neurons resulted in higher excitability and facilitated nociceptive signaling. These results are discussed as leading to an increase in TRPV1 in TG and related satellite cell activation. A large number of satellite cells in TG are known to be activated after tissue inflammation or trigeminal nerve injury (Hanani, 2005). The neuronal activity of TG increased by Nav1.7 causes intracellular ERK phosphorylation, which in turn activates satellite cells around neurons (Black et al., 2012). The activated satellite cells release various molecules, affecting the activity of TG neurons as well as resting satellite cells (Hanani, 2010). This means that pulpitis, which is a result of the inflammatory response of molar 1, consistently induces changes in Nav1.7 that are sufficient to indicate pain in the TG.

In this study, the results of immunostaining and WB data proved that the inflammatory response induced by molar 1 caused not only acute pain but also a chronic increase of Nav1.7 expressions in the TG. The deletion of Nav1.7 (Nav1.7 knockout) in all sensory neurons caused an additional deprivation of noxious thermal sensation (Minett et al., 2012; Kanellopoulos and Matsuyama, 2016). These studies showed the significance of Nav1.7 in pain-related sensations and suggested it as an essential drug target for pain therapies. In the present study, 3 days after pulp inflammation formation, notably increased neuronal activity was detected in the TGs in the AITC group compared to that in the naive and saline-injected groups. Since the AITC group exhibited inflammatory response, pain-related behaviors and increased Nav1.7 expression, it could be concluded that prior results are correlated with the increase in the neuronal excitability of the TG. It has been established that the inflammatory response is sharply reduced when Nav1.7 is knocked out in animal sensory neurons, which are known to be important pathways in the development of inflammatory pain (Nassar et al., 2004; Dib-Hajj et al., 2010). These previous findings are in accordance with our result that the upregulation of neuronal activation in the TGs is related to the increased expression of Nav1.7.

ProTxII, which is extracted from the tarantula, has been proved as a blocker of Nav1.7 (Middleton et al., 2002; Smith et al., 2007). Previous studies have indicated that ProTxII affects inflammation-induced hyperalgesia (Schmalhofer et al., 2008; Emery et al., 2016). In this study, we investigated whether ProTxII inhibits neuronal hyperexcitability by using optical imaging of the TGs in an AITC-induced pulpitis model. We found that ProTxII suppressed excitatory neuronal activity. Moreover, we confirmed that the increased propagation of neuronal activity induced by Nav1.7 expressions in the TGs could be effectively blocked by ProTxII.

Recent animal and clinical studies strongly support that Nav1.7 plays an important role in the pathophysiology of dental disease, such as burning mouth syndrome (Beneng et al., 2010), inflamed TMJ (Bi et al., 2017), trigeminal neuralgia (Zakrzewska et al., 2017), and pulpitis (Aubeux et al., 2021). The high levels of Nav1.7 expressions may be due to pulpitis-induced inflammatory factors and overexpressed interaction of satellite cell-neuron and/or neuron-neuron. The results of our study indicated the inhibition of Nav1.7 channel activities possible clinical application target of pulpitis. Important roles of Nav1.7 in inflammatory pain mechanisms can be a novel target for neuronal excitability, which is related to various pathological circumstances. Although inhibition of Nav1.7 is effective for pain relief, Nav1.7-based drug development for pulpitis-induced pain regulation has not yet been studied. Further research is necessary for the exploitation of Nav1.7 blockers, an upcoming prospective therapeutic strategy for inflammatory pain. Understanding the specific roles of Nav1.7 may contribute to fulfilling the demands for more accurate and effective medicaments for patients with inflammatory pain.

\section{DATA AVAILABILITY STATEMENT}

The original contributions presented in the study are included in the article/Supplementary Material, further inquiries can be directed to the corresponding authors.

\section{ETHICS STATEMENT}

The animal study was reviewed and approved by the Laboratory Animal Facility at Yonsei University.

\section{AUTHOR CONTRIBUTIONS}

MK and MC performed and planned experiments, analyzed and interpreted data, and wrote the article; MC performed surgery, behavioral experiments, and optical imaging; MK contributed to data analysis; and IJ and BHL planned experiments and contributed to writing the article.

\section{FUNDING}

This study was supported by the Basic Research Program through the National Research Foundation (NRF) funded by the Ministry of Science and ICT and Future Planning (NRF2019R1I1A1A01059697 and 2020R1A2C3008481). 


\section{REFERENCES}

Alelyani, A. A., Azar, P. S., Khan, A. A., Chrepa, V., and Diogenes, A. (2020). Quantitative Assessment of Mechanical Allodynia and Central Sensitization in Endodontic Patients. J. Endod. 46, 1841-1848. doi:10.1016/j.joen.2020.09.006

Amir, R., Argoff, C. E., Bennett, G. J., Cummins, T. R., Durieux, M. E., Gerner, P., et al. (2006). The Role of Sodium Channels in Chronic Inflammatory and Neuropathic Pain. J. Pain 7, S1-S29. doi:10.1016/j.jpain.2006.01.444

Aubeux, D., Renard, E., Pérez, F., Tessier, S., Geoffroy, V., and Gaudin, A. (2021). Review of Animal Models to Study Pulp Inflammation. Front. Dental Med. 2, 21. doi:10.3389/fdmed.2021.673552

Beneng, K., Renton, T., Yilmaz, Z., Yiangou, Y., and Anand, P. (2010). Sodium Channel $\mathrm{Na}$ V 1.7 Immunoreactivity in Painful Human Dental Pulp and Burning Mouth Syndrome. BMC Neurosci. 11, 71. doi:10.1186/1471-2202-11-71

Bi, R. Y., Ding, Y., and Gan, Y. H. (2016). Non-Steroidal Anti-Inflammatory Drugs Attenuate Hyperalgesia and Block Upregulation of Trigeminal Ganglionic Sodium Channel 1.7 after Induction of Temporomandibular Joint Inflammation in Rats. Chin. J. Dent Res. 19, 35-42. doi:10.3290/j.cjdr.a35695

Bi, R. Y., Meng, Z., Zhang, P., Wang, X. D., Ding, Y., and Gan, Y. H. (2017). Estradiol Upregulates Voltage-Gated Sodium Channel 1.7 in Trigeminal Ganglion Contributing to Hyperalgesia of Inflamed TMJ. PLoS One 12, e0178589. doi:10.1371/journal.pone.0178589

Black, J. A., Frézel, N., Dib-Hajj, S. D., and Waxman, S. G. (2012). Expression of Nav1.7 in DRG Neurons Extends from Peripheral Terminals in the Skin to central Preterminal Branches and Terminals in the Dorsal Horn. Mol. Pain 8, 82. doi:10.1186/1744-8069-8-82

Black, J. A., Liu, S., Tanaka, M., Cummins, T. R., and Waxman, S. G. (2004). Changes in the Expression of Tetrodotoxin-Sensitive Sodium Channels within Dorsal Root Ganglia Neurons in Inflammatory Pain. Pain 108, 237-247. doi:10.1016/j.pain.2003.12.035

Byers, M. R., and Närhi, M. V. (1999). Dental Injury Models: Experimental Tools for Understanding Neuroinflammatory Interactions and Polymodal Nociceptor Functions. Crit. Rev. Oral Biol. Med. 10, 4-39. doi:10.1177/ 10454411990100010101

Cha, M., Sallem, I., Jang, H. W., and Jung, I. Y. (2020). Role of Transient Receptor Potential Vanilloid Type 1 in the Trigeminal Ganglion and Brain Stem Following Dental Pulp Inflammation. Int. Endod. J. 53, 62-71. doi:10.1111/ iej.13204

Chiang, C. Y., Hu, B., Hu, J. W., Dostrovsky, J. O., and Sessle, B. J. (2002). Central Sensitization of Nociceptive Neurons in Trigeminal Subnucleus Oralis Depends on Integrity of Subnucleus Caudalis. J. Neurophysiol. 88, 256-264. doi:10.1152/ jn.00944.2001

Chiang, C. Y., Zhang, S., Xie, Y. F., Hu, J. W., Dostrovsky, J. O., Salter, M. W., et al. (2005). Endogenous ATP Involvement in Mustard-Oil-Induced Central Sensitization in Trigeminal Subnucleus Caudalis (Medullary Dorsal Horn). J. Neurophysiol. 94, 1751-1760. doi:10.1152/jn.00223.2005

Choi, J. S., Boralevi, F., Brissaud, O., Sánchez-Martín, J., Te Morsche, R. H., DibHajj, S. D., et al. (2011). Paroxysmal Extreme Pain Disorder: A Molecular Lesion of Peripheral Neurons. Nat. Rev. Neurol. 7, 51-55. doi:10.1038/ nrneurol.2010.162

Chudler, E. H., and Byers, M. R. (2005). Behavioural Responses Following Tooth Injury in Rats. Arch. Oral Biol. 50, 333-340. doi:10.1016/ j.archoralbio.2004.08.011

Cummins, T. R., Dib-Hajj, S. D., and Waxman, S. G. (2004). Electrophysiological Properties of Mutant Nav1.7 Sodium Channels in a Painful Inherited Neuropathy. J. Neurosci. 24, 8232-8236. doi:10.1523/JNEUROSCI.269504.2004

Cummins, T. R., Sheets, P. L., and Waxman, S. G. (2007). The Roles of Sodium Channels in Nociception: Implications for Mechanisms of Pain. Pain 131, 243-257. doi:10.1016/j.pain.2007.07.026

Davies, S. L., Loescher, A. R., Clayton, N. M., Bountra, C., Robinson, P. P., and Boissonade, F. M. (2006). Changes in Sodium Channel Expression Following Trigeminal Nerve Injury. Exp. Neurol. 202, 207-216. doi:10.1016/ j.expneurol.2006.05.035

Deuis, J. R., Dvorakova, L. S., and Vetter, I. (2017). Methods Used to Evaluate Pain Behaviors in Rodents. Front. Mol. Neurosci. 10, 284. doi:10.3389/ fnmol.2017.00284
Dib-Hajj, S. D., Cummins, T. R., Black, J. A., and Waxman, S. G. (2007). From Genes to Pain: Na V 1.7 and Human Pain Disorders. Trends Neurosci. 30, 555-563. doi:10.1016/j.tins.2007.08.004

Dib-Hajj, S. D., Cummins, T. R., Black, J. A., and Waxman, S. G. (2010). Sodium Channels in Normal and Pathological Pain. Annu. Rev. Neurosci. 33, 325-347. doi:10.1146/annurev-neuro-060909-153234

Emery, E. C., Luiz, A. P., and Wood, J. N. (2016). Nav1.7 and Other Voltage-Gated Sodium Channels as Drug Targets for Pain Relief. Expert Opin. Ther. Targets 20, 975-983. doi:10.1517/14728222.2016.1162295

Estrela, C., Guedes, O. A., Silva, J. A., Leles, C. R., Estrela, C. R., and Pécora, J. D. (2011). Diagnostic and Clinical Factors Associated with Pulpal and Periapical Pain. Braz. Dent J. 22, 306-311. doi:10.1590/s0103-64402011000400008

Fang, X., Djouhri, L., Black, J. A., Dib-Hajj, S. D., Waxman, S. G., and Lawson, S. N. (2002). The Presence and Role of the Tetrodotoxin-Resistant Sodium Channel $\mathrm{Na}(\mathrm{v}) 1.9(\mathrm{NaN})$ in Nociceptive Primary Afferent Neurons. J. Neurosci. 22, 7425-7433. doi:10.1523/jneurosci.22-17-07425.2002

Filippini, H. F., Scalzilli, P. A., Costa, K. M., Freitas, R. D. S., and Campos, M. M. (2018). Activation of Trigeminal Ganglion Satellite Glial Cells in CFA-Induced Tooth Pulp Pain in Rats. PLoS One 13, e0207411. doi:10.1371/ journal.pone.0207411

Gould, H. J., 3rd, England, J. D., Soignier, R. D., Nolan, P., Minor, L. D., Liu, Z. P., et al. (2004). Ibuprofen Blocks Changes in Na V 1.7 and 1.8 Sodium Channels Associated with Complete Freund's Adjuvant-Induced Inflammation in Rat. J. Pain 5, 270-280. doi:10.1016/j.jpain.2004.04.005

Hameed, S. (2019). Nav1.7 and Nav1.8: Role in the Pathophysiology of Pain. Mol. Pain 15, 1744806919858801. doi:10.1177/1744806919858801

Hanani, M. (2005). Satellite Glial Cells in Sensory Ganglia: From Form to Function. Brain Res. Brain Res. Rev. 48, 457-476. doi:10.1016/j.brainresrev.2004.09.001

Hanani, M. (2010). Satellite Glial Cells in Sympathetic and Parasympathetic Ganglia: In Search of Function. Brain Res. Rev. 64, 304-327. doi:10.1016/ j.brainresrev.2010.04.009

Hargreaves, K. M. (2011). Orofacial Pain. Pain 152, S25-S32. doi:10.1016/ j.pain.2010.12.024

Julius, D., and Basbaum, A. I. (2001). Molecular Mechanisms of Nociception. Nature 413, 203-210. doi:10.1038/35093019

Kanellopoulos, A. H., and Matsuyama, A. (2016). Voltage-gated Sodium Channels and Pain-Related Disorders. Clin. Sci. (Lond) 130, 2257-2265. doi:10.1042/ CS20160041

Kawamura, J., Kaneko, T., Kaneko, M., Sunakawa, M., Kaneko, R., Chokechanachaisakul, U., et al. (2010). Neuron-Immune Interactions in the Sensitized Thalamus Induced by Mustard Oil Application to Rat Molar Pulp. J. Dent Res. 89, 1309-1314. doi:10.1177/0022034510377202

Liu, M., Zhong, J., Xia, L., Dou, N., and Li, S. (2019). The Expression of Voltage-Gated Sodium Channels in Trigeminal Nerve Following Chronic Constriction Injury in Rats. Int. J. Neurosci. 129, 955-962. doi:10.1080/00207454.2019.1595616

Luccarini, P., Childeric, A., Gaydier, A. M., Voisin, D., and Dallel, R. (2006). The Orofacial Formalin Test in the Mouse: A Behavioral Model for Studying Physiology and Modulation of Trigeminal Nociception. J. Pain 7, 908-914. doi:10.1016/j.jpain.2006.04.010

Luiz, A. P., and Wood, J. N. (2016). Sodium Channels in Pain and Cancer: New Therapeutic Opportunities. Adv. Pharmacol. 75, 153-178. doi:10.1016/ bs.apha.2015.12.006

Luo, S., Perry, G. M., Levinson, S. R., and Henry, M. A. (2010). Pulpitis Increases the Proportion of Atypical Nodes of Ranvier in Human Dental Pulp Axons Without a Change in Nav1.6 Sodium Channel Expression. Neuroscience 169, 1881-1887. doi:10.1016/j.neuroscience.2010.06.044

Maarbjerg, S., Di Stefano, G., Bendtsen, L., and Cruccu, G. (2017). Trigeminal Neuralgia - Diagnosis and Treatment. Cephalalgia 37, 648-657. doi:10.1177/ 0333102416687280

Matsuura, S., Shimizu, K., Shinoda, M., Ohara, K., Ogiso, B., Honda, K., et al. (2013). Mechanisms Underlying Ectopic Persistent Tooth-Pulp Pain Following Pulpal Inflammation. PLoS One 8, e52840. doi:10.1371/journal.pone.0052840

Mcdermott, L. A., Weir, G. A., Themistocleous, A. C., Segerdahl, A. R., Blesneac, I., Baskozos, G., et al. (2019). Defining the Functional Role of NaV1.7 in Human Nociception. Neuron 101, 905-919. e8. doi:10.1016/j.neuron.2019.01.047

Middleton, R. E., Warren, V. A., Kraus, R. L., Hwang, J. C., Liu, C. J., Dai, G., et al. (2002). Two Tarantula Peptides Inhibit Activation of Multiple Sodium Channels. Biochemistry 41, 14734-14747. doi:10.1021/bi026546a 
Minett, M. S., Nassar, M. A., Clark, A. K., Passmore, G., Dickenson, A. H., Wang, F., et al. (2012). Distinct Nav1.7-Dependent Pain Sensations Require Different Sets of Sensory and Sympathetic Neurons. Nat. Commun. 3, 791. doi:10.1038/ ncomms 1795

Murano, H., Kaneko, T., Zaw, S. Y. M., Sone, P. P., Zaw, Z. C. T., Okada, Y., et al. (2021). Pulp Inflammation Induces Kv1.1 K(+) Channel Down-Regulation in Rat Thalamus. Oral Dis. [Epub ahead of print]. doi:10.1111/odi.13866

Narita, N., Kumar, N., Cherkas, P. S., Chiang, C. Y., Dostrovsky, J. O., Coderre, T. J., et al. (2012). Systemic Pregabalin Attenuates Sensorimotor Responses and Medullary Glutamate Release in Inflammatory Tooth Pain Model. Neuroscience 218, 359-366. doi:10.1016/j.neuroscience.2012.05.016

Nassar, M. A., Stirling, L. C., Forlani, G., Baker, M. D., Matthews, E. A., Dickenson, A. H., et al. (2004). Nociceptor-Specific Gene Deletion Reveals a Major Role for Nav1.7 (PN1) in Acute and Inflammatory Pain. Proc. Natl. Acad. Sci. U S A. 101, 12706-12711. doi:10.1073/pnas.0404915101

Padilla, F., Couble, M. L., Coste, B., Maingret, F., Clerc, N., Crest, M., et al. (2007). Expression and Localization of the Nav1.9 Sodium Channel in Enteric Neurons and in Trigeminal Sensory Endings: Implication for Intestinal Reflex Function and Orofacial Pain. Mol. Cel Neurosci 35, 138-152. doi:10.1016/ j.mcn.2007.02.008

Rechenberg, D. K., Galicia, J. C., and Peters, O. A. (2016). Biological Markers for Pulpal Inflammation: A Systematic Review. PLoS One 11, e0167289. doi:10.1371/journal.pone.0167289

Rogers, M., Tang, L., Madge, D. J., and Stevens, E. B. (2006). The Role of Sodium Channels in Neuropathic Pain. Semin. Cel Dev Biol 17, 571-581. doi:10.1016/ j.semcdb.2006.10.009

Sangameswaran, L., Fish, L. M., Koch, B. D., Rabert, D. K., Delgado, S. G., Ilnicka, M., et al. (1997). A Novel Tetrodotoxin-Sensitive, Voltage-Gated Sodium Channel Expressed in Rat and Human Dorsal Root Ganglia. J. Biol. Chem. 272, 14805-14809. doi:10.1074/jbc.272.23.14805

Schmalhofer, W. A., Calhoun, J., Burrows, R., Bailey, T., Kohler, M. G., Weinglass, A. B., et al. (2008). ProTx-II, a Selective Inhibitor of NaV1.7 Sodium Channels, Blocks Action Potential Propagation in Nociceptors. Mol. Pharmacol. 74, 1476-1484. doi:10.1124/mol.108.047670

Schuh, C. M. A. P., Benso, B., and Aguayo, S. (2019). Potential Novel Strategies for the Treatment of Dental Pulp-Derived Pain: Pharmacological Approaches and Beyond. Front. Pharmacol. 10, 1068. doi:10.3389/fphar.2019.01068

Sessle, B. J. (2000). Acute and Chronic Craniofacial Pain: Brainstem Mechanisms of Nociceptive Transmission and Neuroplasticity, and Their Clinical Correlates. Crit. Rev. Oral Biol. Med. 11, 57-91. doi:10.1177/10454411000110010401

Siqueira, S. R., Alves, B., Malpartida, H. M., Teixeira, M. J., and Siqueira, J. T. (2009). Abnormal Expression of Voltage-Gated Sodium Channels Nav1.7, Nav1.3 and Nav1.8 in Trigeminal Neuralgia. Neuroscience 164, 573-577. doi:10.1016/j.neuroscience.2009.08.037

Smith, J. J., Cummins, T. R., Alphy, S., and Blumenthal, K. M. (2007). Molecular Interactions of the Gating Modifier Toxin ProTx-II with NaV 1.5: Implied Existence of a Novel Toxin Binding Site Coupled to Activation. J. Biol. Chem. 282, 12687-12697. doi:10.1074/jbc.M610462200

Sooratgar, A., Ahmadi, Z., Asadi, Y., Dibaji, F., Shamshiri, A. R., and Afkhami, F. (2021). Evaluation of Secondary Thermal Hyperalgesia Resulting from Pulpal Inflammation in Patients with Symptomatic Irreversible Pulpitis. J. Endod. 47, 902-905. doi:10.1016/j.joen.2021.02.010

Sperry, M. M., Yu, Y. H., Welch, R. L., Granquist, E. J., and Winkelstein, B. A. (2018). Grading Facial Expression Is a Sensitive Means to Detect Grimace Differences in Orofacial Pain in a Rat Model. Sci. Rep. 8, 13894. doi:10.1038/ s41598-018-32297-2
Strickland, I. T., Martindale, J. C., Woodhams, P. L., Reeve, A. J., Chessell, I. P., and Mcqueen, D. S. (2008). Changes in the Expression of NaV1.7, NaV1.8 and NaV1.9 in a Distinct Population of Dorsal Root Ganglia Innervating the Rat Knee Joint in a Model of Chronic Inflammatory Joint Pain. Eur. J. Pain 12, 564-572. doi:10.1016/j.ejpain.2007.09.001

Sun, S., Sun, J., Jiang, W., Wang, W., and Ni, L. (2019). Nav1.7 via Promotion of ERK in the Trigeminal Ganglion Plays an Important Role in the Induction of Pulpitis Inflammatory Pain. Biomed. Res. Int. 2019, 6973932. doi:10.1155/2019/ 6973932

Toledo-Aral, J. J., Moss, B. L., He, Z. J., Koszowski, A. G., Whisenand, T., Levinson, S. R., et al. (1997). Identification of PN1, a Predominant Voltage-Dependent Sodium Channel Expressed Principally in Peripheral Neurons. Proc. Natl. Acad. Sci. U S A. 94, 1527-1532. doi:10.1073/pnas.94.4.1527

Tsuboi, Y., Iwata, K., Dostrovsky, J. O., Chiang, C. Y., Sessle, B. J., and Hu, J. W. (2011). Modulation of Astroglial Glutamine Synthetase Activity Affects Nociceptive Behaviour and Central Sensitization of Medullary Dorsal Horn Nociceptive Neurons in a Rat Model of Chronic Pulpitis. Eur. J. Neurosci. 34, 292-302. doi:10.1111/j.1460-9568.2011.07747.x

Vos, B. P., Hans, G., and Adriaensen, H. (1998). Behavioral Assessment of Facial Pain in Rats: Face Grooming Patterns after Painful and Non-Painful Sensory Disturbances in the Territory of the Rat's Infraorbital Nerve. Pain 76, 173-178. doi:10.1016/s0304-3959(98)00039-6

Wood, J. N., Boorman, J. P., Okuse, K., and Baker, M. D. (2004). Voltage-Gated Sodium Channels and Pain Pathways. J. Neurobiol. 61, 55-71. doi:10.1002/ neu. 20094

Yeomans, D. C., Levinson, S. R., Peters, M. C., Koszowski, A. G., Tzabazis, A. Z., Gilly, W. F., et al. (2005). Decrease in Inflammatory Hyperalgesia by Herpes Vector-Mediated Knockdown of Nav1.7 Sodium Channels in Primary Afferents. Hum. Gene Ther. 16, 271-277. doi:10.1089/hum.2005.16.271

Zakrzewska, J. M., Palmer, J., Morisset, V., Giblin, G. M., Obermann, M., Ettlin, D. A., et al. (2017). Safety and Efficacy of a Nav1.7 Selective Sodium Channel Blocker in Patients with Trigeminal Neuralgia: A Double-Blind, PlaceboControlled, Randomised Withdrawal Phase 2a Trial. Lancet Neurol. 16, 291-300. doi:10.1016/S1474-4422(17)30005-4

Zhang, P., and Gan, Y. H. (2017). Prostaglandin E2 Upregulated Trigeminal Ganglionic Sodium Channel 1.7 Involving Temporomandibular Joint Inflammatory Pain in Rats. Inflammation 40, 1102-1109. doi:10.1007/ s10753-017-0552-2

Conflict of Interest: The authors declare that the research was conducted in the absence of any commercial or financial relationships that could be construed as a potential conflict of interest.

Publisher's Note: All claims expressed in this article are solely those of the authors and do not necessarily represent those of their affiliated organizations, or those of the publisher, the editors and the reviewers. Any product that may be evaluated in this article, or claim that may be made by its manufacturer, is not guaranteed or endorsed by the publisher.

Copyright (c) 2021 Kwon, Jung, Cha and Lee. This is an open-access article distributed under the terms of the Creative Commons Attribution License (CC $B Y)$. The use, distribution or reproduction in other forums is permitted, provided the original author(s) and the copyright owner(s) are credited and that the original publication in this journal is cited, in accordance with accepted academic practice. No use, distribution or reproduction is permitted which does not comply with these terms. 